\title{
A unified approach to remeshing strategies for finite element $h$-adaptivity
}

\author{
Pedro Díez, Antonio Huerta* \\ Departament de Matemàtica Aplicada III, E.T.S. Ingenieros de Caminos, Universitat Politècnica de Catalunya, E-08034 Barcelona, \\ Spain
}

\begin{abstract}
In $h$-adaptivity, a remeshing strategy is needed to compute the distribution of required element size using the estimated error distribution. Several authors have introduced different remeshing strategies yielding very different results. In this work these methods are included in a unified framework, emphasizing the role of the underlying hypotheses. Moreover, an objective tool to evaluate the accuracy of the resulting finite element solution is presented. Thus, a new remeshing strategy is introduced to optimize the accuracy of the adapted solutions. The different remeshing strategies are compared with well-known numerical examples. .
\end{abstract}

\section{Introduction}

Adaptive computations are used to obtain finite element results with a controlled accuracy. The scheme of an adaptive procedure is shown in Fig. 1. The domain is first discretized with an initial guess of the optimal mesh. Then, the discretization is enriched using the information given by an error estimate and the solution is gradually improved until it is accurate enough.

Srategies based on $h$-adaptivity, see for instance $[1-4,6,7,9-12,16]$, consist on building a new mesh, using the same type of elements, and 'adapting' the element size to the requirements of the solution. That is, reducing their size where the interpolation must be enriched (i.e. more accuracy is needed) and enlarging the elements where it is already accurate enough. The number of degrees of freedom is increased (or decreased) in every zone of the computational domain in order to obtain some prescribed accuracy. An error assessment is needed to design a new mesh verifying the accuracy prescriptions. The numerical tool used in the error assessment may be either an error estimator or an error indicator.

In fact, adaptive computations can be viewed as an iterative procedure that generates a convergent sequence of approximations to the exact solution. It is important to notice that convergence is a key issue in adaptive computations that must be ensured in every step of the scheme depicted in Fig. 1. The finite element model must be convergent (i.e. a priori error estimates must exist). The optimality criterion, see Fig. 1, which is used to generate the new mesh must be coherent with the a priori error estimates. And the a posteriori error estimator (or error indicator) must be in accordance with the equations. That is, error estimators relying on the ellipticity of the equations cannot be used in hyperbolic problems, see, for instance, $[13,4]$ for a discussion in the context of localization problems.

There is a general consensus on the convergence criterion that must be employed to stop this iterative procedure: the so-called acceptability criterion presented in next section. However, there are several criteria

*Corresponding author. 


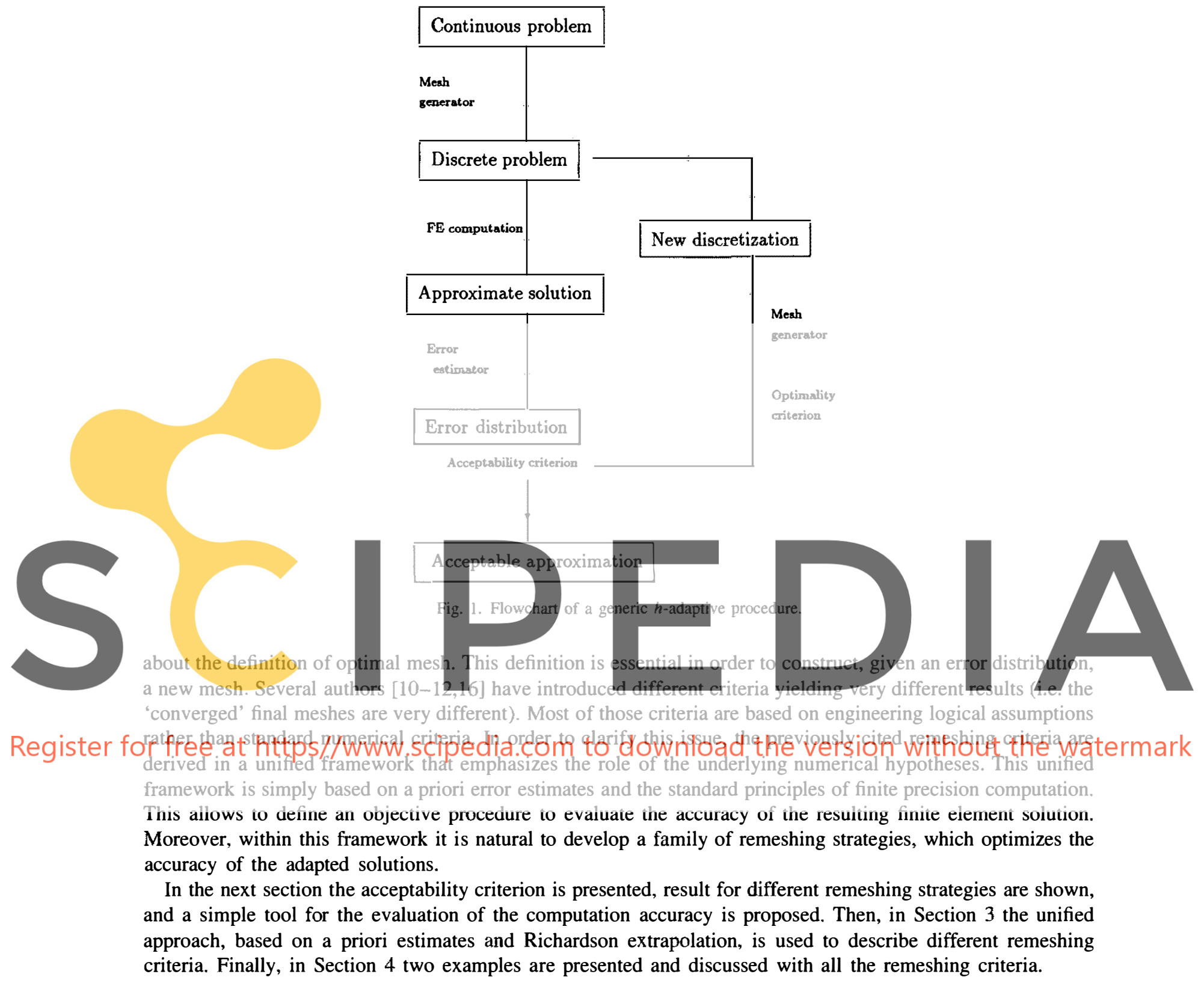

\section{Main ingredients of $h$-adaptivity}

\subsection{Acceptability criterion}

The use of $h$-adaptivity generates a sequence of meshes that are expected to converge to a proper mesh. In order to carry out practical computations this sequence must be stopped and, consequently, an acceptability criterion must be defined. The acceptability criterion sets the threshold of the prescribed computational accuracy. If the acceptability criterion is satisfied, the solution is accurate enough and, therefore, the adaptive procedure is stopped. If it is not satisfied, a new mesh must be constructed.

A standard acceptability criterion prescribes the global error measured in energy norm, that is

$$
\|e\| \leq \eta\|u\|
$$


where $u$ is the exact solution, $e$ is the error (difference between the exact solution and the approximation, $u_{h}$ ), and $\eta$ is the prescribed relative error.

REMARK 1 . The expression of the energy norm introduced above is associated with the bilinear form, say $a(\cdot)$, of the weak formulation of the problem:

$$
\|e\|:=\sqrt{a(e, e)}:=\left[\int_{\Omega} \nabla e \cdot K \cdot \nabla e \mathrm{~d} \Omega\right]^{1 / 2},
$$

where the right-hand side term illustrates a typical expression for $a(\cdot, \cdot)$ corresponding to a diffusion equation with conductivity tensor $\boldsymbol{K}$.

The acceptability criterion introduced in Eq. (1) is based on global considerations because the measures of $e$ and $u$ concern the complete computational domain $\Omega$. However, if a new mesh must be created, local information regarding the desired element size is required. Thus, new criteria must be introduced in order to define the optimai distribution of the element size in the domain.

\subsection{Remeshing strategy}

$h$-adaptive computations based on different error assessment techniques are common in the literature $[1,3,9-12,16]$. Although all of them use the acceptability criterion described in Eq. (1) and the same, or equivalent, error estimators, the resulting meshes are very different. For instance, if the remeshing strategies introduced by Zienkiewiqz and Zhu [16], Li and Bettes [11], and Oñate and Bugeda [12] are usqd in a test case (elastic plane strain analysi estimator [3], the final ines elements differ. Neverthele below the threshold of the remeshing strategies leading to mesh is an optimal mesh.
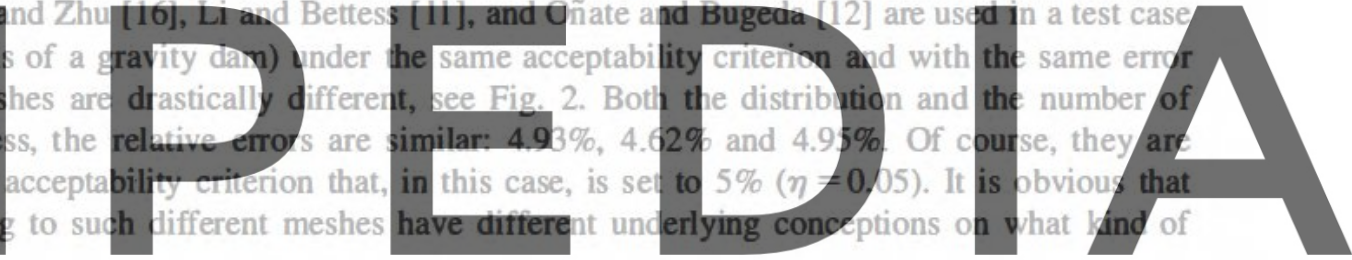

A remeshing strategy is, actually, a formula giving the desired element size in the domain as a function of the

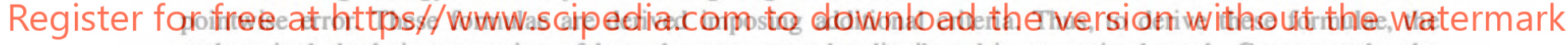
authors include their conception of how the error must be distributed in an optimal mesh. Consequently, the assumed additional prescriptions to the final error distribution are called optimality criteria.

The remainder of this section is devoted to define a tool to assess the quality of the obtained solutions. Thus, the efficiency of different optimality criteria can be evaluated. The next section presents remeshing strategies related to different optimality criteria introduced under a unified approach.

\subsection{Computation accuracy}

The accuracy of a numerical approximation is associated with the absolute and relative errors. In scientific computation, however, it is the relative error that is of interest because the results may vary enormously in magnitude. The relative error is a precise measure of the accuracy, which is base independent. The notion of correct significant digits (and their mathematically consistent definition) is directly related to the relative error. Namely, $\bar{x}$ agrees with $x$ to $c$ significant digits if $|x-\bar{x}|$ is less than half a unit in the cth significant digit of $x$, see an excellent presentation in [5, Chap. 1]. This definition implies the following expression

$$
\left|\frac{x-\bar{x}}{x}\right| \leq \frac{1}{2} 10^{-c}
$$

when $x$ is non-zero, which can be extended to

$$
|\bar{x}| \leq \frac{1}{2} 10^{-c}
$$

if $x=0$. For instance, $\bar{x}=0.003$ has two correct significant digits of zero but not three; $\bar{x}=0.3210$ agrees to $x=0.3230$ to only one significant digit but $\bar{x}=0.3210$ agrees to $x=0.3195$ to two significant digits. 


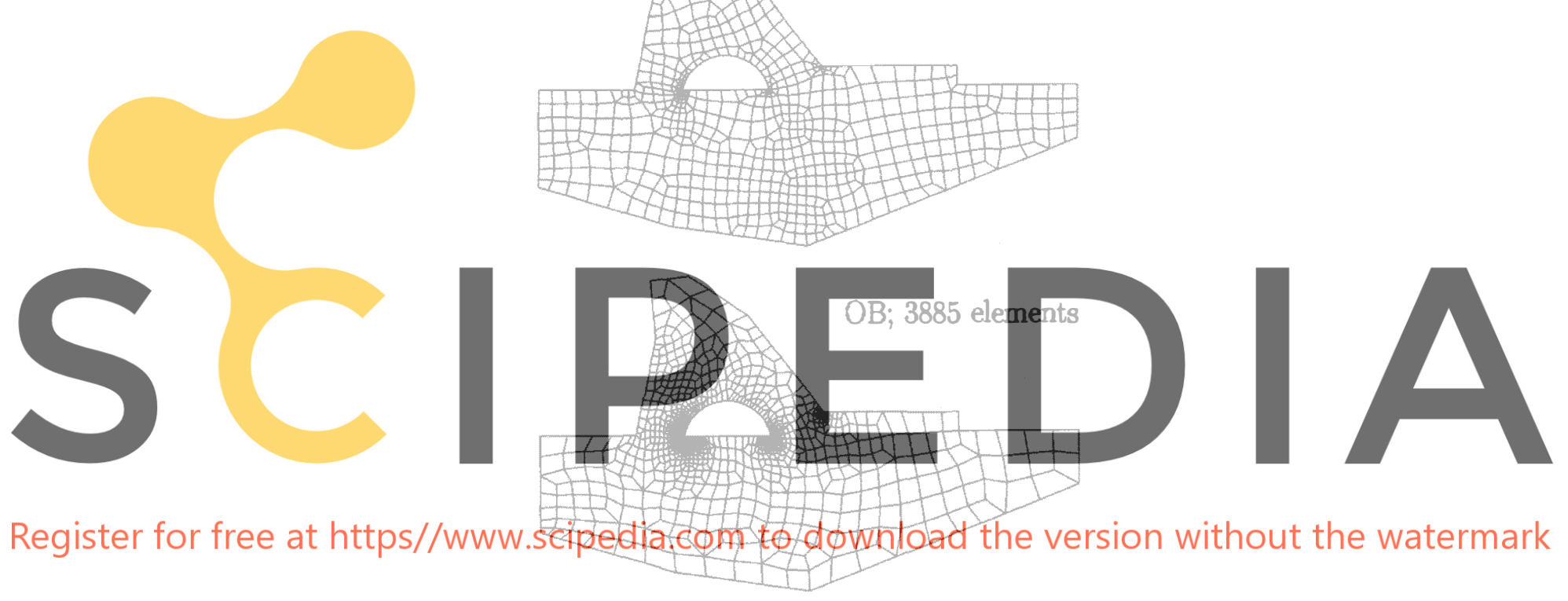

Fig. 2. Finai meshes obtained with different optimaitity criteria.

When $x$ and $\bar{x}$ are vectors the relative errors are often defined with global norms (Euclidean, maximum or Manhattan). However, it is also very useful to treat every component independently. If the componentwise relative error [5], is used in the previous equations one can ensure $c$ significant digits for every component of the vector.

The above considerations can be applied to measure the local quality of the finite element solution. However, in this case, the approximated quantities are functions and not numbers. Consequently, the absolute values of Eqs. (3) and (4) must be replaced by a functional norm, typically the energy norm. Moreover, if a componentwise relative error is used, instead of the global norm (over the entire domain), local restrictions (over each element) are employed to ensure $c$ significant digits for every element of the discretized domain.

Denoting by $\|\cdot\|_{k}$ the restriction of the energy norm to the element $\Omega_{k}$ of the mesh $(k=1, \ldots, M)$, the accuracy of the solution in the element $\Omega_{k}$ is described by $\|e\|_{k}$ (which plays the role of $|x-\bar{x}|$ ) and $\|u\|_{k}$ (which plays the role of $|x|)$. However, the exact solution $u$ is unknown. Nevertheless, using Galerkin finite elements, $u_{h}$ can be seen as a projection of $u$ and, therefore, $u_{h}$ and $e$ are orthogonal. This orthogonality is assumed to stand also at the local level and, consequently, $\|u\|_{k}$ is approximated using $V\left\|u_{h}\right\|_{k}^{2}+\|e\|_{k}^{2}$. Thus, the number of correct significant digits in the element $\Omega_{k}$ is

$$
c=-\log _{10}\left(2 \frac{\|e\|_{k}}{\sqrt{\left\|u_{h}\right\|_{k}^{2}+\|e\|_{k}^{2}}}\right) .
$$

The value of $c$ computed using Eq. (5) is not an integer, but it is a meaningful measure of the accuracy. 


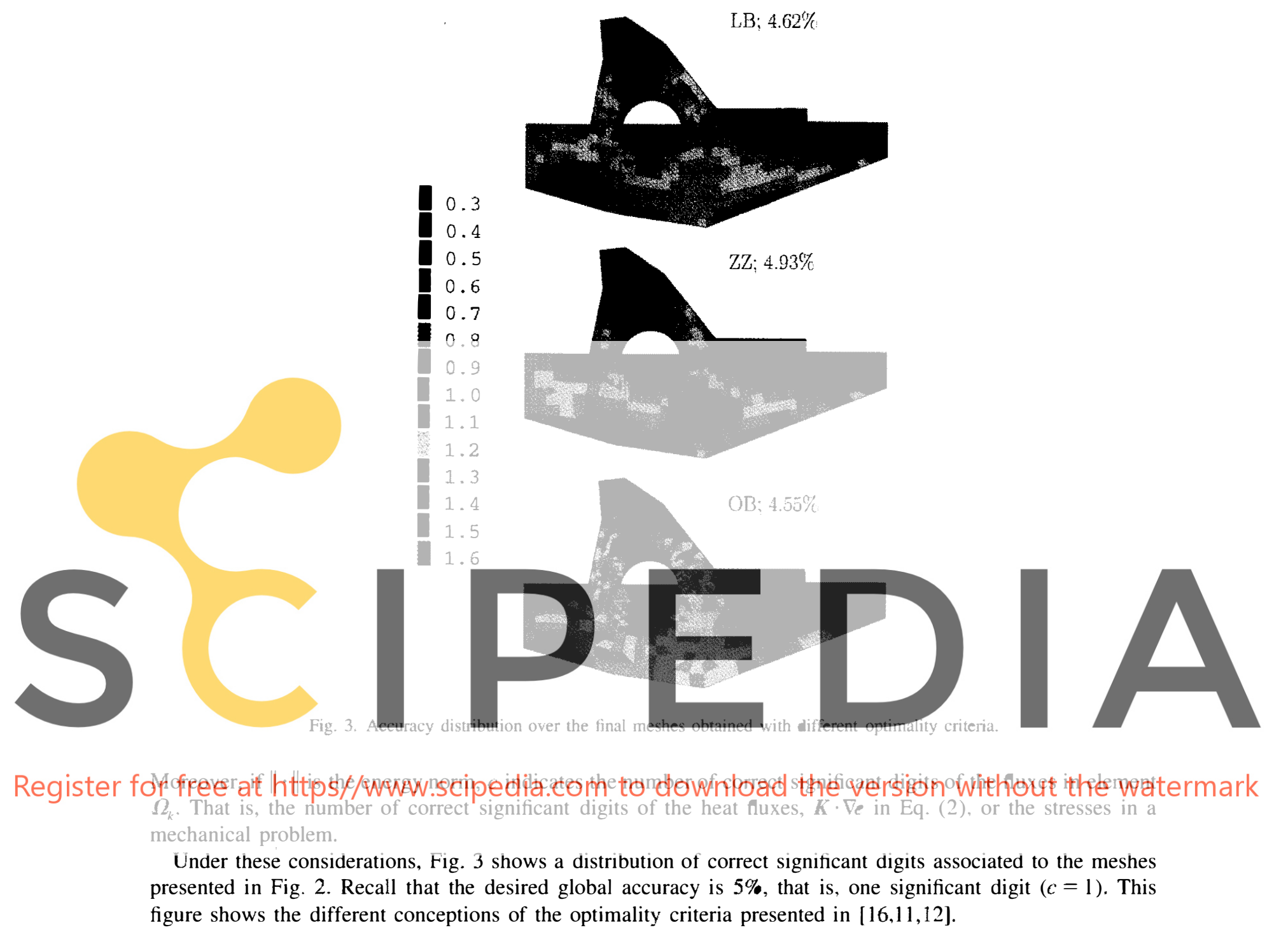

\section{Optimality criteria and asseciated remeshing strategies}

\subsection{A priori estimates and Richardson extrapolation}

Different remeshing criteria may be derived in a general approach applying Richardson extrapolation to the a priori error estimates of the finite element solutions. An a priori estimate for an elliptic problem stands [8]

$$
\|e\| \leq \alpha h^{m},
$$

where $m$ is related with the maximum degree of the complete polynomials included in the interpolation. In fact, for second-order elliptic problems $m$ is equal to the degree of the interpolating polynomials. The constant $\alpha$ is independent of the element size $h$ but it is unknown. $\alpha$ depends on the distortion of the elements and it is proportional to a measure of the $m+1$ derivatives of function $u\left(\alpha=\alpha^{*}|u|_{m+1, \Omega}\right)$. Thus

$$
\|e\| \leq\left.\left.\alpha^{*}\right|_{u}\right|_{m+1, \Omega^{2}} h^{m}
$$

where $|\cdot|_{m+1, \Omega}$ is the seminorm in $H^{m+1}(\Omega)$.

Eq. (7) is the fundamental error estimate for the elliptic boundary-value problem [8, p. 189]. It represents, 
however, a global measure, where $h$ is a characteristic element size (the diameter of the largest element in the mesh). In order to develop optimality criteria based on mathematical hypotheses, a local a priori error estimate is needed. That is, a particularization of the previous formula over one element. In fact, a direct particularization of Eq. (7) over element $k$ is possible if pollution errors are negligible. Local optimality of the Galerkin finite element solution, $\|e\|_{k} \leq\left\|u \quad v_{h}\right\|_{k} \forall v_{h} \in H^{m+1}\left(\Omega_{k}\right)$, is necessary to obtain the a priori estimate [8]. This local optimality condition stands if pollution errors are negligible [15]. In fact, in adaptive computations for elliptic problems, the influence of pollution errors becomes negligible as the adaptive process progresses, see for instance the examples shown in [7]. Under this hypothesis a local a priori error estimate describing the asymptotic behavior of the local error norm in each element can be written as

$$
\|e\|_{k} \leq \alpha_{k} h_{k}^{m}
$$

where $\alpha_{k}$ is proportional to a local measure of the $m+1$ derivatives of $u\left(\alpha_{k}=\alpha^{*}|u|_{m+1, \Omega_{k}}\right)$. Due to the fact that $\alpha_{k}$ depends on $h_{k}$ through $|u|_{m+1, \Omega_{k}}$

$$
\|e\|_{k} \leq \alpha^{*}|u|_{m+1 . \Omega_{k}} h_{k}^{m}
$$

is not, as shown, a suitable local error bound; an extra step is needed.

In elliptic problems it seems reasonable to assume that the $m+1$ derivatives of $u$ in $\Omega$ are regular enough; then it can be stated that the local seminorm is bounded by the global one,

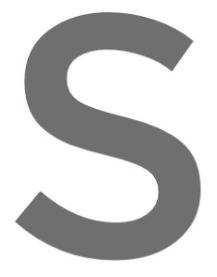

$$
|u|_{m+1 \Omega_{k}} \leq \beta \frac{\Omega_{k}^{1 / 2}}{\Omega^{1 / 2}}|u|_{m+1, \Omega}
$$

\section{where the areas $\Omega_{k}$ an} $\bar{\alpha}=\alpha^{*} \beta / \Omega^{1 / 2}$, a local

\section{where $h_{k}$ is a characteristic size o}
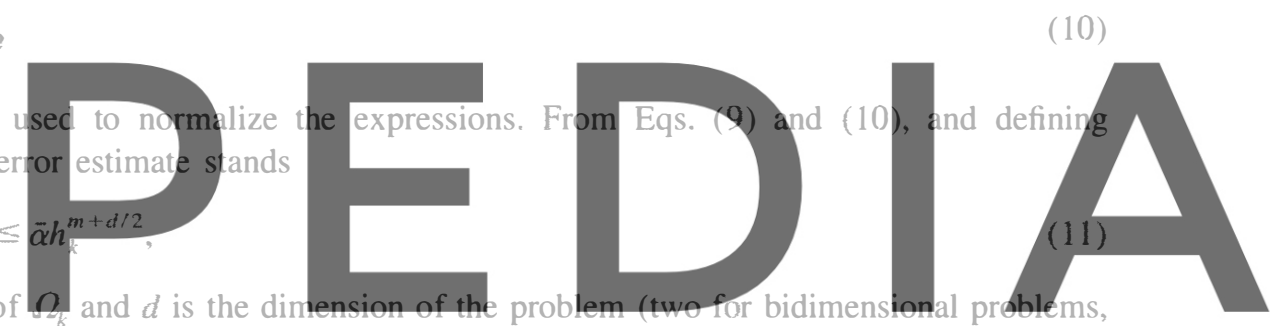

three for tridimensional ones). The two alternative versions for local priori estimates in Eq. (11) are equivalent if $h_{k} \approx \Omega_{k}^{1 / d}$.

Standard Richardson extrapolation applied to Eq. (6) allows to predict the characteristic element size $\hat{h}$ of a new mesh that ensures the prescribed accuracy $\hat{e}$, namely,

$$
\hat{h}=\left[\frac{\|\hat{e}\|}{\|e\|}\right]^{1 / m} h \text {. }
$$

Note that everything associated with the new mesh (the one that has to be generated) is denoted with a hat.

This is again a characteristic size of the complete new mesh. However, the goal of the remeshing strategy is to prescribe the element size as a function of the pointwise error. That is, to derive an expression for the element size of the new mesh inside every element of the old one. If $\hat{h}_{k}$ denotes the desired element size within element $\Omega_{k}$, expressions for $\hat{h}_{k}$ analogous to Eq. (13) are derived using the local a priori estimates of Eq. (11):

$$
\hat{h}_{k}=h_{k}\left[\frac{\|\hat{e}\|_{k} \Omega_{k}^{1 / 2}}{\|e\|_{k} \hat{\Omega}_{k}^{1 / 2}}\right]^{1 / m} \quad \text { or } \quad \hat{h}_{k}=h_{k}\left[\frac{\|\hat{e}\|_{k}}{\|e\|_{k}}\right]^{1 /(m+d / 2)}
$$

where index $\hat{k}$ refers to an element of the new mesh, $\hat{\Omega}_{\widehat{k}}$ is the area of a new element, and $\|\cdot\|_{\mathcal{K}}$ indicates the restriction of the energy norm to element $\hat{k}$ of the new mesh. Nevertheless, Eq. (14) is determined for each element $\Omega_{k}$ of the old mesh. Thus, in Eq. (14) the index $\hat{k}$ does not range between 1 and $\hat{M}(\hat{M}$ is the total number of elements in the new mesh), it only refers to those new elements (i.e. those $\hat{k}$ ) lying inside element $\Omega_{k}$ of the old mesh.

In order to obtain practical expressions of the remeshing criteria, the value of $\|\hat{e}\|_{\hat{k}}$ must be prescribed in Eq. 
(14). This is done using an optimality criterion. Using different optimality criteria, Eq. (14) is particularized in the following well-known remeshing strategies.

\subsection{Li and Bettess remeshing strategy (LB)}

This remeshing strategy $[1 \mathbf{0}, 11]$ is based on considering that the error distribution in an optimal mesh is uniform, that is

$$
\|\hat{e}\|_{k}=\frac{\|\hat{e}\|}{\sqrt{\hat{M}}}=\frac{\eta\|\| \|}{\sqrt{\hat{M}}} \text { for } \hat{k}=1, \ldots, \hat{M} .
$$

Replacing Eq. (15) in Eq. (14),

$$
\hat{h}_{k}=\left[\frac{\eta\|d\|}{\sqrt{\hat{M}}\|l\|_{k}}\right]^{1 /(m+d / 2)} h_{k}
$$

Although the number of elements in the new mesh, $\hat{M}$, is unknown, it can be a priori approximated using the assumption of Eq. (12). The number of elements of the new mesh lying inside the element $\Omega_{k}$ of the original mesh is $\left[h_{k} / \hat{h}_{k}\right]^{d}$. Then, a new expression for $\hat{M}$ is obtained:

$$
\hat{M}=\sum_{k=1}^{M}\left[\frac{h_{k}}{\hat{h}_{k}}\right]^{d}
$$

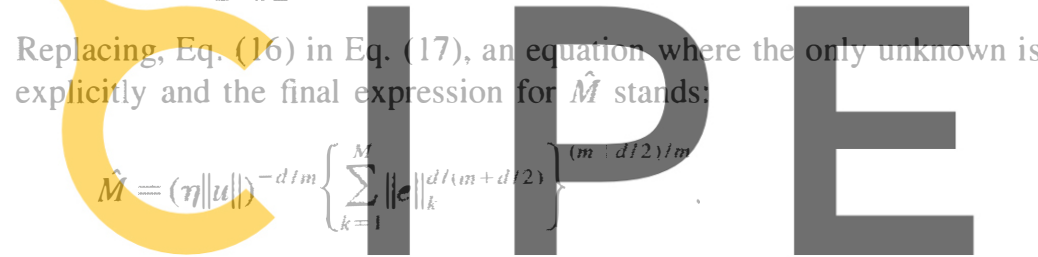

This remeshing criterion has been proven, see [10], to be optimal in the sense that, of all methods verifying the (17)

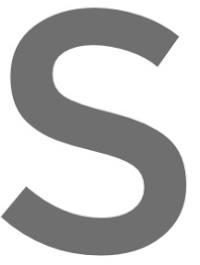

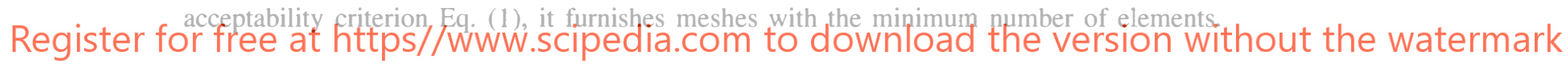

\subsection{Zienkiewicz and Zhu remeshing strategy (ZZ)}

This strategy [16] can be seen as a simplified version of the previous one. In this case, the mesh is said to be optimal if the error distribution in the new mesh is uniform referred to the elements of the old mesh, that is

$$
\|\hat{e}\|_{k}=\frac{\|\hat{e}\|}{\sqrt{M}}=\frac{\eta\|\imath\|}{\sqrt{ } M} \text { før } k=1, \ldots, M \text {. }
$$

An additional assumption is needed: the density of energy associated to the error in the new mesh is constant inside each element $\Omega_{k}$ of the old mesh, that is

$$
\frac{\|\hat{e}\|_{k}}{\hat{\Omega}_{k}^{1 / 2}}=\frac{\|\hat{e}\|_{k}}{\Omega_{k}^{1 / 2}} \text {. }
$$

With this hypothesis the $\mathrm{ZZ}$ criterion is obtained [16],

$$
\hat{h}_{k}=\left[\frac{\eta\|u\|}{\sqrt{M}\|e\|_{k}}\right]_{h_{k}}^{1 / m} .
$$

\subsection{Oñate and Bugeda remeshing strategy $(O B)$}

The previous remeshing strategies are based on optimality criteria that impose uniform distributions of the local measures of the error. Consequently, the induced remeshing formulae are such that elements with the same amount of error are equally subdivided, regardless of their size. However, engineering intuition seems to 
indicate that the elements must be refined according to the density of error. In [12] a criterion which enforces a uniform error density is proposed, that is

$$
\frac{\|\hat{e}\|_{k}}{\hat{\Omega}_{k}^{1 / 2}}=\frac{\|\hat{e}\|}{\Omega^{1 / 2}}=\frac{\eta\|u\|}{\Omega^{1 / 2}} \text { for } \hat{k}=1, \ldots, \hat{M}
$$

Thus, substituting in Eq. (14), the desired element size is obtained

$$
\hat{h}_{k}=\left[\frac{\eta\|u\| \Omega_{k}^{1 / 2}}{\|e\|_{k} \Omega^{1 / 2}}\right]^{1 / m} h_{k}
$$

\subsection{Remeshing strategy enforcing Uniform Local Accuracy (ULA)}

All the previously presented criteria enforce, as optimal, a value of the error (or error density). In fact, from a numerical point of view, these criteria impose an absolute error. However, it is now standard to measure accuracy with relative error, which does not depend on the variations of magnitude and it is base independent. If an analogy is established with convergence criteria in iterative methods for linear systems of equations, the previously described criteria are equivalent to seeking a constant absolute error for each component of the solution vector. This is not a standard practice in numerical methods. Usually, either a global measure of the relative error is employed, such as the acceptability criterion defined in Eq. (1), or a componentwise relative error is enforced.

Although LB is objectively 'optimal' (in the sense that leads to cheaper meshes), the uniformity of the error distribution has been prescr only objective tool availab as defined in Eq. (5). However, that relative er

The usual numerical criterion is b approximate zero, namely.

$$
\|\hat{e}\|_{k}^{2}=\eta_{\mathrm{L}}^{2}\|u\|_{\hat{R}}^{2}+E_{\mathrm{A}}^{2}
$$
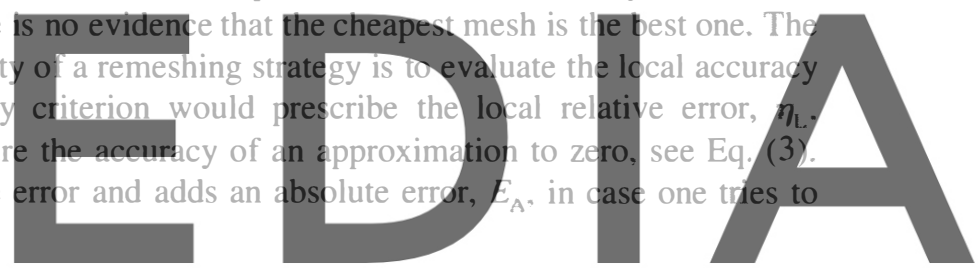

(24)

Register for free at https//www.scipedia, com to download the version without the watermark

The value of the absolute error, $E_{\mathrm{A}}$, may be arbitrarily chosen. Here, $E_{\mathrm{A}}$ is defined in order to relate in a simple way the parameters in Eq. (24), $\eta_{\mathrm{L}}$ and $E_{\mathrm{A}}$, with the parameter $\eta$ in the acceptabihity criterion, Eq. (1). $E_{\mathrm{A}}$ is defined as the absolute error one would get in each element if the global relative error is $\eta_{\mathrm{A}}$ and the error density is uniform over the entire domain. That is,

$$
E_{\mathrm{A}}=\eta_{\mathrm{A}} \frac{\|u\|}{\Omega^{1 / 2}} \hat{\Omega}_{\hat{k}}^{1 / 2}
$$

In fact, summing up Eq. (24) for every $\hat{k}$ after substitution of Eq. (25), the global error measure is directly related to the energy norm of the solution, $\|\hat{e}\|^{2}=\left(\eta_{\mathrm{L}}^{2}+\eta_{\mathrm{A}}^{2}\right)\|\iota\|^{2}$, and a simple relation between $\eta$, $\eta_{\mathrm{I}}$ and $\eta_{\mathrm{A}}$ is obtained

$$
\eta=\sqrt{\eta_{\mathrm{L}}^{2}+\eta_{\mathrm{A}}^{2}}
$$

Eq. (24) may replace the acceptability criterion of Eq. (1). The solution may be considered acceptable if every local error is lower than the right-hand side of Eq. (24). However, this acceptability criterion is very restrictive and yields final meshes with a large number of elements. Thus, the acceptability criterion of Eq. (1) is still used to verify convergence and Eq. (24) suffices to set the remeshing strategy and it is not strictly enforced.

Assuming that the energy of the solution $u$ is uniform inside each element $\Omega_{k}$, the ULA criterion, Eq. (24) after substitution of (25), stands

$$
\frac{\|\hat{e}\|_{k}^{2}}{\hat{\Omega}_{\hat{k}}}=\eta_{\mathrm{L}}^{2} \frac{\|u\|_{k}^{2}}{\Omega_{k}}+\eta_{\mathrm{A}}^{2} \frac{\|u\|^{2}}{\Omega} .
$$


Replacing Eq. (27) in Eq. (14), a remeshing formula is found:

$$
\hat{h}_{k}=\left[\frac{\Omega_{k}^{1 / 2}}{\|e\|_{k}} \sqrt{\eta_{\mathrm{L}}^{2} \frac{\|u\|_{k}^{2}}{\Omega_{k}}+\eta_{\mathrm{A}}^{2} \frac{\|u\|^{2}}{\Omega}}\right]^{1 / m} h_{k} .
$$

It is worth noting that this remeshing strategy seeks for meshes with a local accuracy prescribed by $\eta_{L}$ and a local absolute error $E_{\mathrm{A}}$ associated with a global relative error $\eta_{\mathrm{A}}$. In fact, the absolute error $E_{\mathrm{A}}$ plays a role only if the local norm of $u,\|u\|_{k}$, is very small.

Whenever Eq. (26) is satisfied, the results obtained with the ULA remeshing strategy with parameters $\eta_{\mathrm{L}}$ and $\eta_{\mathrm{A}}$ may be compared with the results of the previous strategies with parameter $\eta$. The possibility of handling two parameters allows the user to tune the remeshing strategy depending on the problem. For instance, if one sets $\eta_{\mathrm{L}}=\eta$ and $\eta_{\mathrm{A}}=0$, the criterion is purely relative and the zones with zero (or almost) energy are unlikely refined. On the contrary, if $\eta_{\mathrm{L}}=0$ and $\eta_{\mathrm{A}}=\eta$, the ULA strategy is exactly the same as the LB strategy. The main feature of the ULA strategy is to use a proper combination of the two parameters. As it is shown in the next section, numerical experiments have demonstrated that the global relative error, $\eta$, must be split in a local relative error, $\eta_{\mathrm{L}}$, which takes most of it $\left(\eta_{\mathrm{L}} \approx 0.98 \eta\right)$ and an absolute error associated with $\eta_{\mathrm{A}}$, which is clearly relaxed compared with the $\mathrm{LB}$ criterion $\left(\eta_{\mathrm{A}} \approx 0.20 \eta\right)$.

\section{Numerical examples}

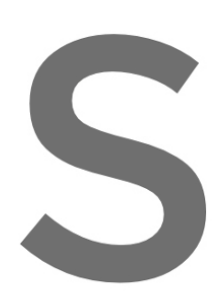

The behavior of different is a standard linear elastic introduced $[16,12]$. The cross section is discretize into account the respons The second example allows to test the behavi concentrated) and zones with very low strain energy.

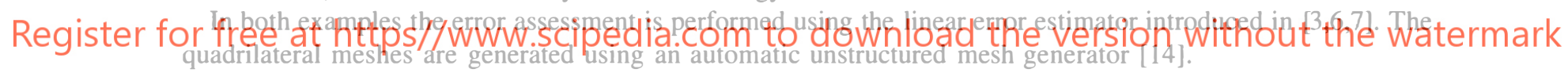

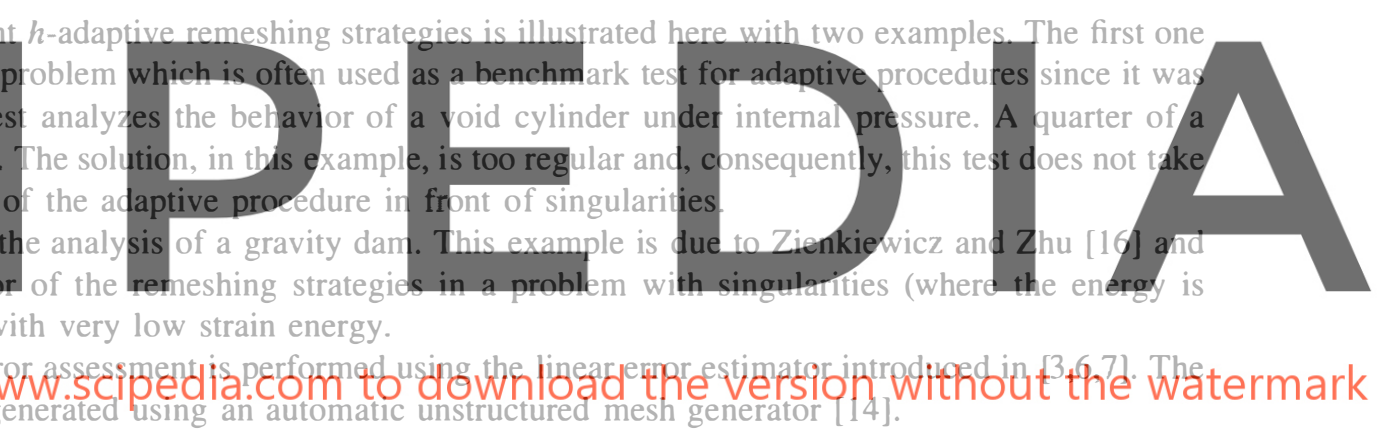

\subsection{Example 1: thick circular cylinder under internai pressure}

In this example the required error $\eta$ in the acceptability criterion of Eq. (1) is set to 5\%, that is, the expected value of the accuracy is $c=1$. The adaptive analysis is performed using different remeshing strategies: $\mathrm{LB}, \mathrm{ZZ}$, OB and ULA for $\eta_{\mathrm{L}}=5 \%\left(\eta_{\mathrm{A}}=0\right), \eta_{\mathrm{L}}=4.9 \%\left(\eta_{\mathrm{A}} \approx 1 \%\right), \eta_{\mathrm{L}}=4 \%\left(\eta_{\mathrm{A}}=3 \%\right), \eta_{\mathrm{L}}=3 \%\left(\eta_{\mathrm{A}}=4 \%\right)$ and $\eta_{\mathrm{L}}=1 \%\left(\eta_{\mathrm{A}} \approx 4.9 \%\right)$ (the corresponding values of $\eta_{\mathrm{A}}$ are taken such that Eq. (26) is verified). All the remeshing procedures are started by the original roughly uniform mesh shown in the center of Fig. 4. As it has been noticed in [12] the $\mathrm{ZZ}$ remeshing strategy applied to this problem oscillates, due to alternative refinement and derefinement of some mesh zones. In order to obtain an acceptable solution, the $\mathrm{ZZ}$ remeshing strategy is modified in the sense that derefinement is not allowed. Then an admissible solution is found after only one remeshing step but the error is $3.23 \%$, much below the required 5\%, and the number of elements is much larger than expected. The rest of the remeshing strategies converge to admissible solutions in less than three remeshing steps. The results are summarized in Table 1 which shows, for each remeshing strategy, the number of remeshing steps needed to reach an acceptable mesh, the number of elements and the global error.

Fig. 4 shows the final mesh obtained with every remeshing strategy and the distribution of the correct significant digits, $c$, defined in Eq. (5). It can be seen how the distribution of $c$ is almost uniform for the ULA strategy with $\eta_{\mathrm{L}}=5 \%$. That is, the fully relative local optimality criterion leads to a uniform accuracy in the domain. Notice that in this case, the obtained mesh is almost structured. The LB strategy produces similar results with slightly less elements and a not so uniform accuracy distribution. The different ULA strategies generated decreasing the parameter $\eta_{\mathrm{L}}$ from $5 \%$ to $0 \%$ (that is, approaching the OB strategy) yield results that are scaled in a gradual transition. The limit case of the OB strategy produces a mesh with a large number of 


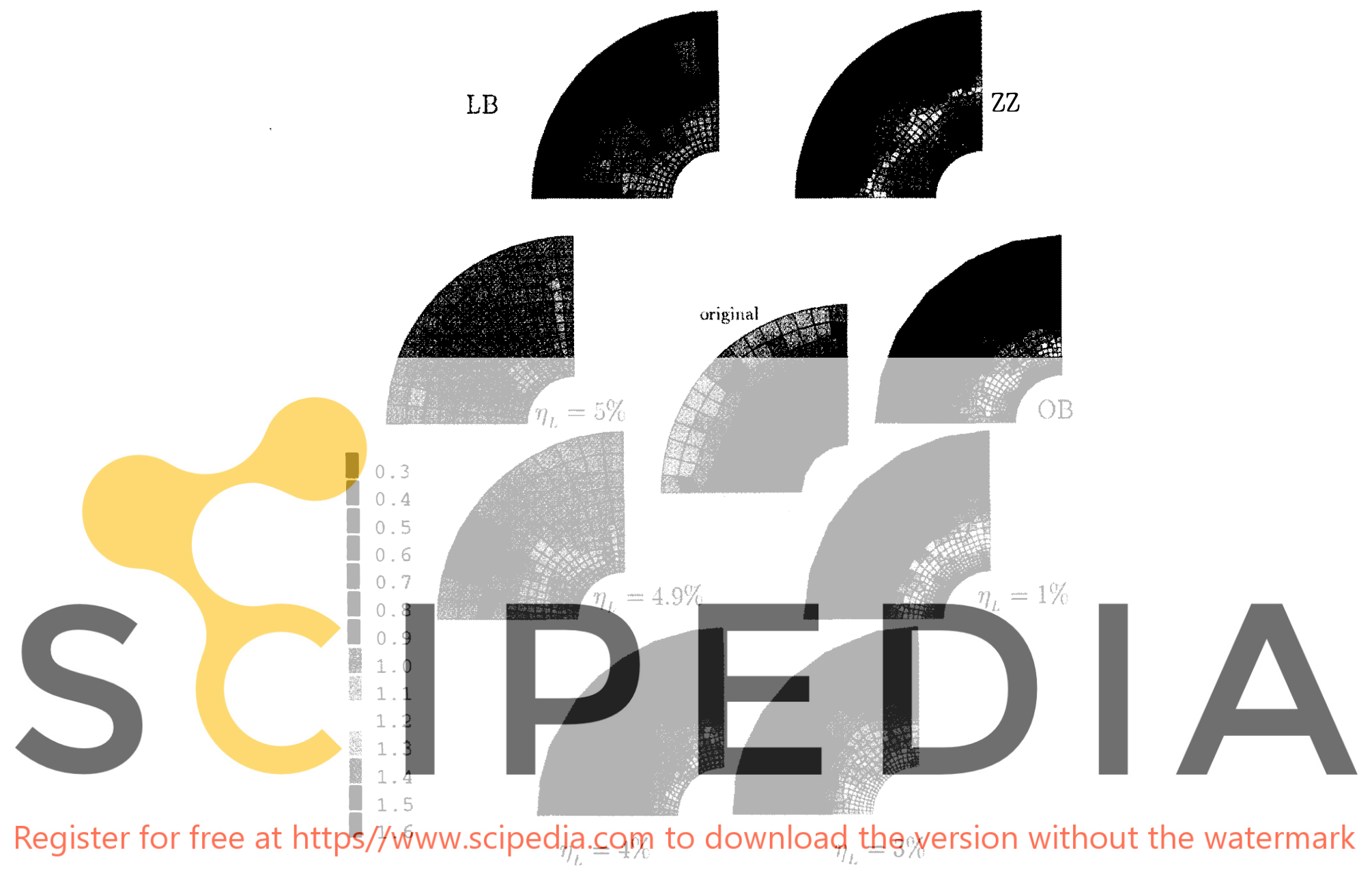

Fig. 4. Example 1: Final mesh of the adaptive procedure using different remeshing strategies. The color scale indicates the accuracy distribution.

Table 1

Summary of the results for Examples 1 and 2

\begin{tabular}{|c|c|c|c|c|c|c|}
\hline \multirow[t]{2}{*}{ Remeshing strategy } & \multicolumn{3}{|l|}{ Example 1} & \multicolumn{3}{|l|}{ Example 2} \\
\hline & num. steps & num. elts. & $\%$ error & num. steps & num. elts. & $\%$ erro \\
\hline Original mesh & - & 84 & $14.54 \%$ & $=$ & 193 & $11.48 \%$ \\
\hline LB & 1 & 169 & $4.94 \%$ & 2 & 685 & $4.62 \%$ \\
\hline $\mathrm{ZZ}$ & 1 & 608 & $3.23 \%$ & 4 & 602 & $4.93 \%$ \\
\hline OB & 2 & 537 & $4.99 \%$ & 3 & 3885 & $4.55 \%$ \\
\hline ULA $\eta_{\mathrm{L}}=1 \%$ & 3 & 470 & $4.93 \%$ & 2 & 1007 & $4.95 \%$ \\
\hline ULA $\eta_{1}=3 \%$ & 3 & 239 & $4.85 \%$ & 3 & 1492 & $4.70 \%$ \\
\hline LLA $\eta_{\mathrm{l}}=4 \%$ & 2 & 174 & $4.95 \%$ & 3 & 1548 & $4.94 \%$ \\
\hline LLA $\eta_{\mathrm{L}}=4.9 \%$ & 2 & 200 & $4.94 \%$ & 4 & 3497 & $5.11 \%$ \\
\hline ULA $\eta_{\mathrm{L}}=5 \%$ & 3 & 194 & $4.84 \%$ & 2 & 3226 & $4.51 \%$ \\
\hline
\end{tabular}

elements and a sharp accuracy distribution. The accuracy is high where elements are small (in the interior part of the cylinder) but it is too low in the rest of the domain. As previously said, the $\mathrm{ZZ}$ strategy does not perform well in this test (it oscillates) and its modified version produces a mesh with too many elements but some zones with insufficient accuracy. 
In order to give a graphic representation of the uniformity of the accuracy distribution in the domain, Fig. 5 shows a set of histograms, one for every mesh in Fig. 4. Each histogram plots the accumulated element area versus $c$. That is, the height of the histogram bar is the sum of the areas of the elements having the corresponding accuracy $c$. Since the expected value of $c$ is 1 , the ideal situation is to obtain a histogram concentrated in 1 (one bar with height equal to the total area of the domain). This representation confirms that the ULA method with $\eta_{\mathrm{L}}=5 \%$ has, in this case, a very good behavior. The second best is the ULA method with $\eta_{\mathrm{L}}=4.9 \%$ and third is the LB strategy; they both show a clear peak near one. The rest of the strategies do not behave that well.
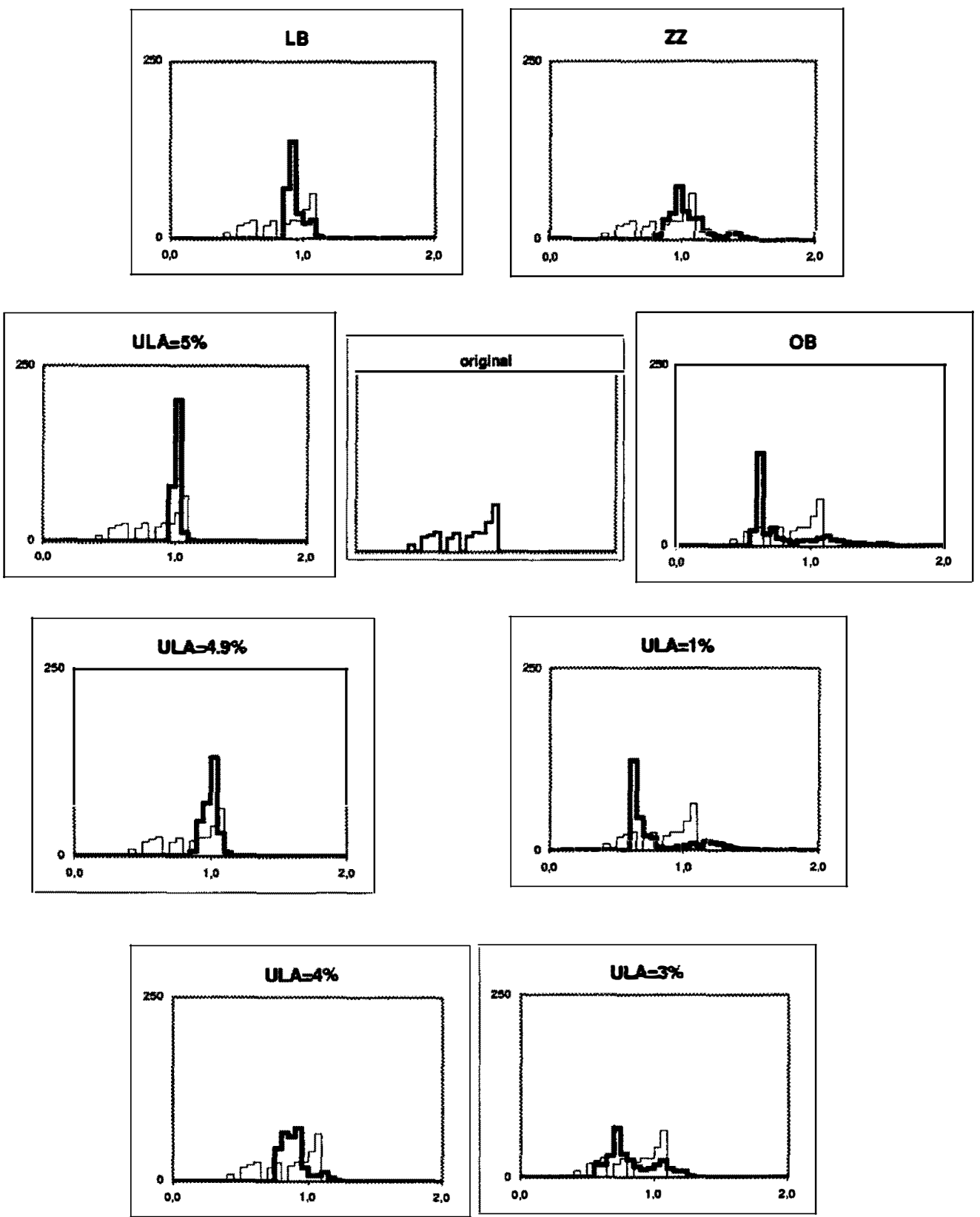

Fig. 5. Example 1: histogram of the area corresponding to every value of the accuracy (described by $c$ ). The histograms correspond to the final meshes obtained with different remeshing strategies and are compared with the histogram associated with the original mesh. 


\subsection{Example 2: plane strain analysis of a dam}

Starting from the original mesh in the center of Fig. 6, a set of mesh sequences is generated using different remeshing strategies. The prescribed accuracy for the acceptability criterion of Eq. (1) is also set to $\eta=5 \%$ $(c=1)$.

The same strategies compared in the previous example are used here. The results are also summarized in Table 1. Fig. 6 shows the final meshes obtained with each remeshing strategy. All the variations of ULA strategy and the $O B$ strategy (which can be regarded as a particular case of the ULA with $\eta_{L}=\mathbf{0}$ ) yield meshes with a much larger number of elements than the $\mathrm{ZZ}$ and LB remeshing strategies. Of course, the global accuracy is similar in all the cases because they all use the same acceptability criterion, Eq. (1).

Nevertheless, the distribution of the local accuracy reveals that the quality of the solutions is indeed very different. Fig. 7 shows the distribution of the local magnitude $c$ defined in Eq. (5) for all the meshes of Fig. 6.

It must be noted that $\mathrm{ZZ}$ and LB strategies yield meshes with local errors far below the desired accuracy in areas of the domain which are interesting from an engineering viewpoint (around the cavity, and in particular at

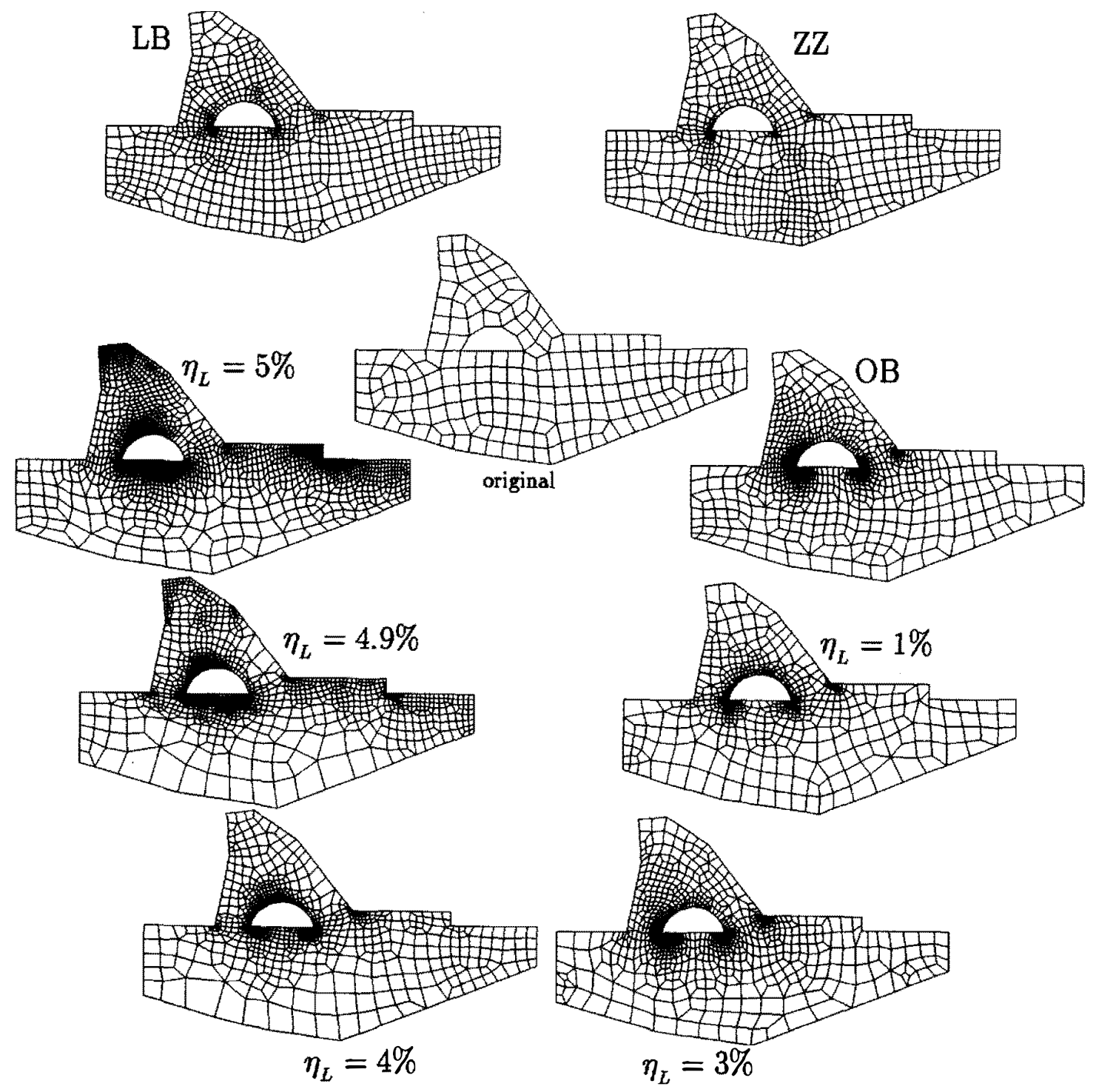

Fig. 6. Example 2: Final mesh of the adaptive procedure using different remeshing strategies. 


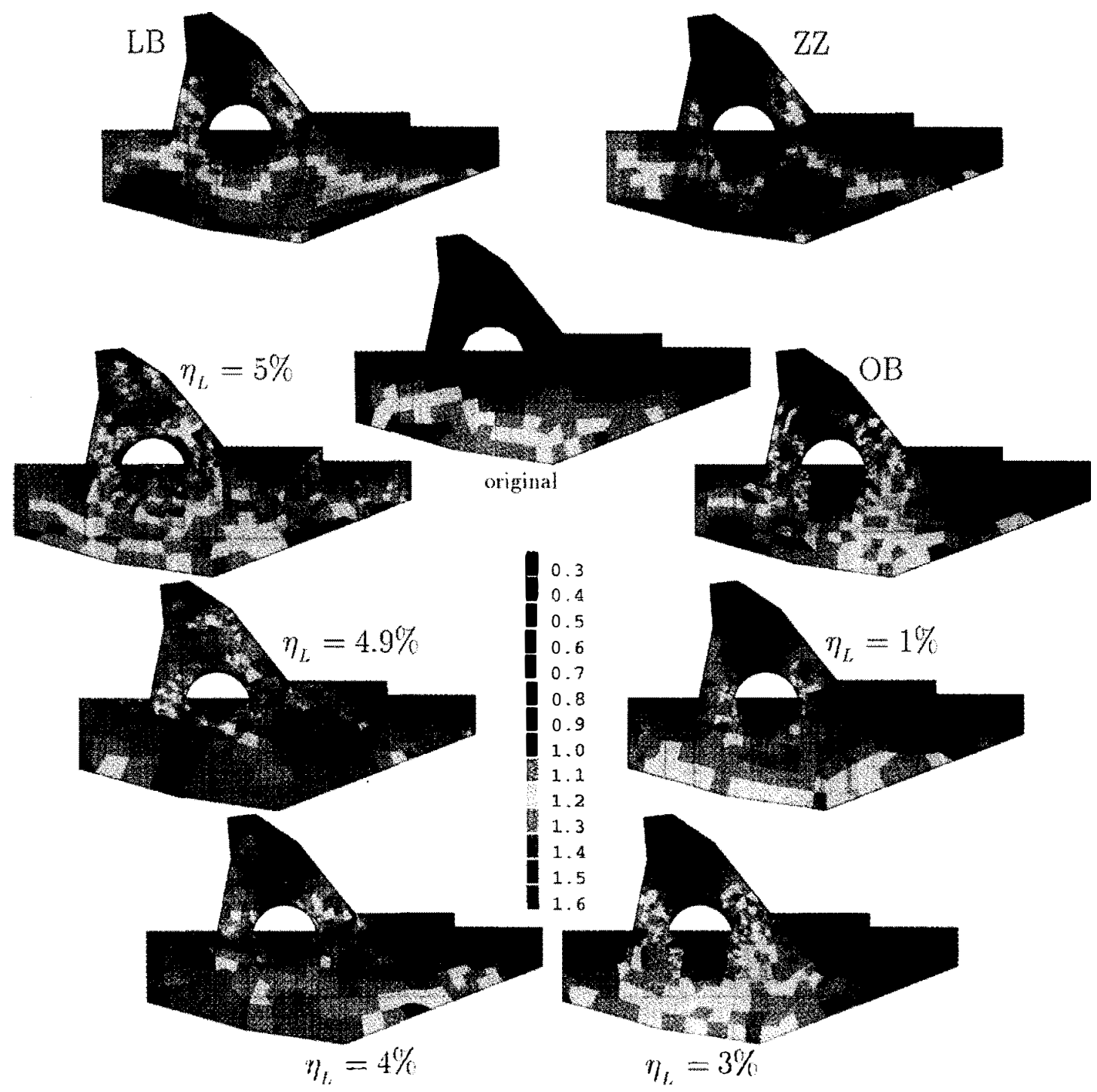

Fig. 7. Example 2: accuracy distribution for every remeshing strategy.

the corners). In fact, even the OB strategy which introduces enough elements at the corners is also far below the desired accuracy in the rest of the cavity.

The ULA criterion yields a family of results. The mesh obtained with the purely local criterion $\left(\eta_{\mathrm{L}}=5 \%\right.$ and $\eta_{\mathrm{A}}=0$ ) concentrates elements in zones where the solution has almost zero energy, that is where the displacement is mainly a rigid body motion and strains and stresses are very small. Of course, from a practical point of view, this solution is too expensive. The introduction of parameter $\eta_{\mathrm{A}}$ solves this problem allowing the local error to be equal to the local absolute value $E_{\mathrm{A}}$, see Eq. (24). And as soon as $\eta_{\mathrm{A}} \neq 0$ the distribution of elements seems, as expected, more reasonable from a practical point of view. Now, for $\eta_{\mathrm{L}}=4.9 \%$ the accuracy distribution near the cavity is the desired one. In fact, even for $\eta_{\mathrm{L}}=4 \%$ the distribution of $c$ near the cavity is superior to the $\mathrm{ZZ}, \mathrm{LB}$ and $\mathrm{OB}$ strategies. In fact, these two examples also outperform the others when the uniformity of the accuracy distribution in the domain is analyzed, Fig. 8. If computer cost is a limiting condition, the ULA criterion with $\eta_{\mathrm{L}}=4 \%$ seems the better choice. In this case, the local accuracy is satisfied, at least in the critical zones, and the accuracy distribution is quite uniform. 

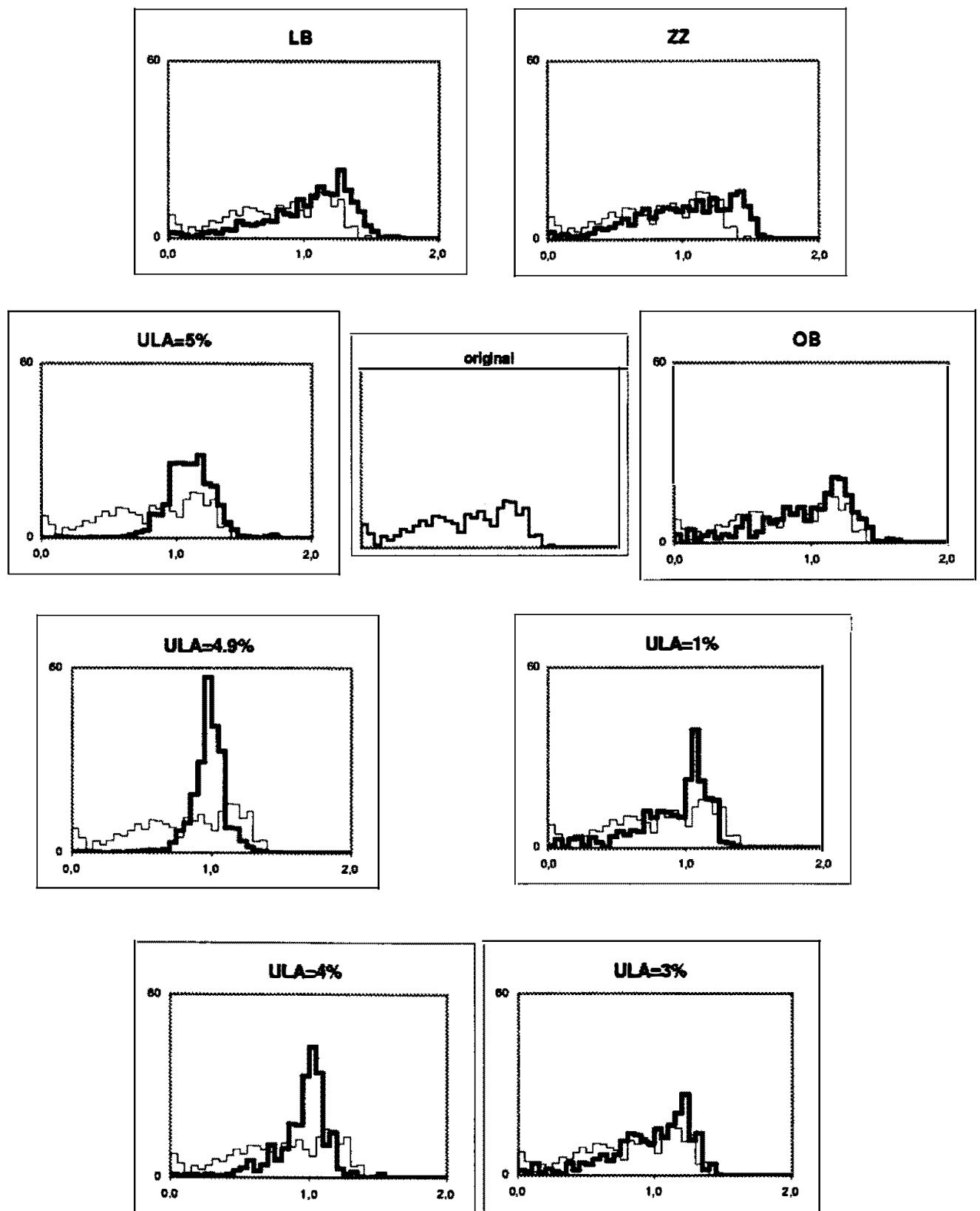

Fig. 8. Example 2: histogram of the area corresponding to every value of the accuracy (described by $c$ ). The histograms correspond to the final meshes obtained with different remeshing strategies and are compared with the histogram associated with the original mesh.

\section{Concluding remarks}

A unified approach to remeshing strategies for finite element $h$-adaptivity is presented. It is based on a priori error estimates and the standard principles of finite precision computation. This allows to define an objective procedure to evaluate the local accuracy of the resulting finite element solution. This tool is used to assess the quality of the different solutions induced by different remeshing criteria. Moreover, within this framework it is natural to develop a new family of remeshing strategies, which optimizes the local accuracy of the adapted solutions. Numerical examples demonstrate that the quality of the obtained solution depends on the remeshing strategy. The obtained accuracy is strongly related with the underlying optimality criterion that generates each remeshing strategy. 


\section{References}

[1] M. Ainsworth and J.T. Oden, A unified approach to a posteriori error estimation using element residual methods, Numer. Math. 65 (1993) 23-50.

[2] F. Cirak and E. Ramm, A posteriori error estimation and adaptivity for linear elasticity using the reciprocal theorem, Comput. Methods Appl. Mech. Engrg. 156 (1998) 351362.

[3] P. Díez, J.J. Egozcue and A. Huerta, A posteriori error estimation for standard finite element analysis, Comput. Methods Appl. Mech. Engrg. 163 (1998) 141157.

[4] P. Díez, M. Arroyo and A. Huerta, Adaptivity based on error estimation for viscoelastic softening materials, Mechanics of Cohesive-Frictional Materials (to be published).

[5] N. Higham, Accuracy and stability of numerical algorithms, Society for Industrial and Applied Mathematics (SIAM, Philadelphia, PA, 1996).

[6] A. Huerta, P. Díez and J.J. Egozcue, Error estimation for linear and nonlinear problems, in: M. Křížek et al., eds., Finite Element Methods: Superconvergence, Post-processing and a Posteriori Error Estimates (Marcel Dekker, New York, 1997) 183194.

[7] A. Huerta and P. Díez, Error estimation including pollution assessment for nonlinear finite element analysis, Comput. Methods Appl. Mech. Engrg. in press.

[8] T.J.R. Hughes, The Finite Element Method (Prentice Hall International, Stanford, 1987).

[9] $\mathrm{P}$. Ladevèze and $\mathrm{Ph}$. Rougeot, New advances on a posteriori error on constitutive relation in f.e. analysis, Comput. Methods Appl. Mech. Engrg. 150 (1997) 239249.

[10] L.Y. Li, P. Bettess, J.W. Bull, T. Bond and I. Applegarth, Theoretical formulations for adaptive finite element computations, Comm. Numer. Methods Engrg. 11 (1995) 857868

[11] L.Y. Li and P. Bettess, Notes on mesh optimal criteria in adaptive finite element computations, Comm. Numer. Methods Engrg. 11 (1995) 911915.

[12] E. Oñate and G. Bugeda, A study of mesh optimality criteria in adaptive finite element analysis, Engrg. Comput. 10 (1993) 307321.

[13] M. Ortiz and J.J. Quigley IV, Adaptive mesh refinement in strain localization problems, Comput. Methods Appl. Mech. Engrg. 90 (1991) 781804

[14] J. Sarrate, Modelización numérica de la interacción fluido-sólido rígido: desarrollo de algoritmos, generación de mallas y adaptividad, Doctoral Thesis, Universitat Politècnica de Catalunya, Barcelona, 1996.

[15] L.B. Wahlbin, Local behavior in finite element methods, in: P.G. Ciarlet and J.L. Lions, eds., Handbook of Numerical Analysis, Vol. II (North Holland, Amsterdam, 1991) 357522.

[16] O.C. Zienkiewicz and J.Z. Zhu, A simple error estimator and adaptive procedure for practical engineering analysis, Int. J. Numer. Methods Engrg. 24 (1987) 337357. 